\title{
Improving the Depth and Accuracy of HAZOP Analysis for Safer Process Development in Chemical Industries
}

\author{
Jingyi Li \\ School of Engineering, Stanford University, Palo Alto, United States
}

Email address:

lirache131@gmail.com

To cite this article:

Jingyi Li. Improving the Depth and Accuracy of HAZOP Analysis for Safer Process Development in Chemical Industries. American Journal of Chemical Engineering. Vol. 9, No. 1, 2021, pp. 18-24. doi: 10.11648/j.ajche.20210901.12

Received: January 11, 2021; Accepted: January 18, 2021; Published: February 10, 2021

\begin{abstract}
HAZOP analysis has become a versatile tool for industrial risk assessment and optimization in the past decades. It facilitates systematical design review with wide applications spanning across entire project lifecycle, from initial design to operation and decommission stages. Traditional qualitative HAZOP process that largely depends on historical experience and brainstorming can lead to inaccurate hazard identification and severe accident consequences. This study aims at improving the depth and accuracy of HAZOP analysis by delivering a comprehensive exploration of the critical factors and advanced quantitative approach. The impact factors were illustrated from prerequisite and assurance aspects. Prerequisite factors serve as the fundamentals of HAZOP which involve design technical details, HAZOP team management, execution strategy and HSE culture, while assurance factors denote the systematical PSI data and quantitative analytical frameworks. A classical chemical case study via semi-quantitative method was exemplified. Countermeasures and international leading practices were introduced with a summary chart at the end. Special attention should be paid to the effectiveness of safety guards and coming up HAZOP recommendations. Motivating future works can be explored such as HAZOP efficiency optimization, finer study targeting different project types, and broader industry applications. By incorporating the critical factors with integrated quantitative approach, the influence of enterprise HAZOP analysis will be more profound with enhanced accident prevention and risk awareness in the overall industrial environment.
\end{abstract}

Keywords: HAZard and OPerability (HAZOP) Analysis, Process Safety, Risk Management, Risk Matrix, Enterprise HSE, Chemical Engineering

\section{Introduction}

\subsection{Background}

HAZOP analysis (Hazard and Operability Analysis) is a safety assurance technique predominantly applied in chemical engineering industry to identify the potential hazards, root causes, protective measures together with long-term preventions. It is a systematical team activity facilitated by HAZOP leader and multidisciplinary professionals, involving rigorous review of design documents and control schemes [1]. With half century of development since its inception in 1960s, HAZOP technique has now evolved into a powerful tool for industrial risk assessment and prioritization comparing with traditional methods. It has been used for diverse industries such as petrochemicals, pharmaceuticals, oil and gas, and nuclear fields, spanning the entire project life cycle from initial design stage, through operation and modification, to decommissioning at the end [2].

The core significance of HAZOP analysis is through early process screening and rectification to address potential hazards and deliver facilities with improved safety and operability.

Nowadays, HAZOP technique has become one of the systematical design review methods led by enterprises to ensure plant intrinsic safety and process safety $[3,4]$. From risk management standpoint, HAZOP scenarios can be consolidated into the integrated risk matrix of an enterprise [4] and used as invaluable training materials to strengthen long-term risk management database.

\subsection{Common Issues in HAZOP Analysis}

As process risk reduction gets more emphasized in many 
countries through legislation in recent years, HAZOP technique is becoming more universal and versatile, yet with evident issues as below for improvement:

Incomplete hazard identification, such as insufficient evaluation of root causes, severity and probability of the consequences.

Unclear identification of different levels of protection layers and their impacts, causing ineffective HAZOP solutions and recommendations.

Failure to reveal process intrinsic limitations, such as historical hazards or unknown ones.

As a result, corrective proposals from HAZOP analysis in many scenarios tend to be relatively simple and cannot tackle the problem effectively. In other words, the depth and accuracy of HAZOP approach are not meeting the realistic demands $[5,6]$. In addition, an industrial HAZOP process typically takes huge time and labor investment due to the detailed design reviews and cross-functional participation [7, 8]. Therefore, it is an imperative topic to improve the HAZOP approach in order to maximize the efficiency and applicability for various industries.

This article presents a comprehensive exploration on the critical impact factors from both prerequisite and assurance levels, supported with an industrial case study. Optimized methodology and solutions are proposed as countermeasures. Although HAZOP process has been widely discussed, there were few literatures focusing on the systematical research of HAZOP quality. This paper showcases a pilot study originated from chemical industries while the conclusions can be extended to other applicable fields.

The study is organized as follows: Section 1 introduces the theme and commons issues in today's HAZOP applications. Section 2 and 3 present an innovative analysis on the critical factors and a semi-quantitative example with proposed solutions. An overall summary of key takeaways and the special issues for attention are illustrated in Section 4 and 5 to conclude this study.

\section{Analysis of Prerequisite Factors}

The "prerequisites factors" fall into three areas: technical conditions preparation, HAZOP team management, execution strategy and corporate HSE culture. These factors are the objective fundamentals to deliver HAZOP outcomes with sufficient depth and accuracy.

\subsection{Technical Conditions Preparation}

Technical conditions [9] such as design completion status, process or plant technical schemes, and P\&ID drawings can be concisely called as process safety information (PSI). They are the technical prerequisites and the most basic conditions for a thorough and accurate HAZOP analysis.

HAZOP process is highly dependent on design documents as the main data source. However, there are common cases that people tend to rush the HAZOP analysis schedule in spite of premature technical schemes and incomplete P\&ID, especially for revamp projects or modified processes.
HAZOP in this case, is more like a regulatory step rather than an in-depth study with practical effects. There would not be fixed equipment configuration and material selection for an accurate risk evaluation before completing the technical schemes.

The right strategy is to develop an overall plan and ensure necessary PSI in place before initiating any HAZOP activities. It is hereby a good practice to review P\&ID designs in advance to ensure full compliance with industry standards and project required functions. Finally, it is recommended to submit all the technical preparations one week earlier in order to identify any gaps and allow the team to get familiar before the formal HAZOP process.

\subsection{HAZOP Team Management}

Establishing a professional HAZOP team with strong leadership, in-depth expertise, and effective management serves as the essential foundation for successful HAZOP outcomes.

HAZOP leadership is one of the critical factors determining the quality of each HAZOP activity. A HAZOP leader needs comprehensive expertise in plant operation, PSM (process safety management), industry-specific knowledge, and strong communication skills. A HAZOP leadership competency model was developed in Figure 1.

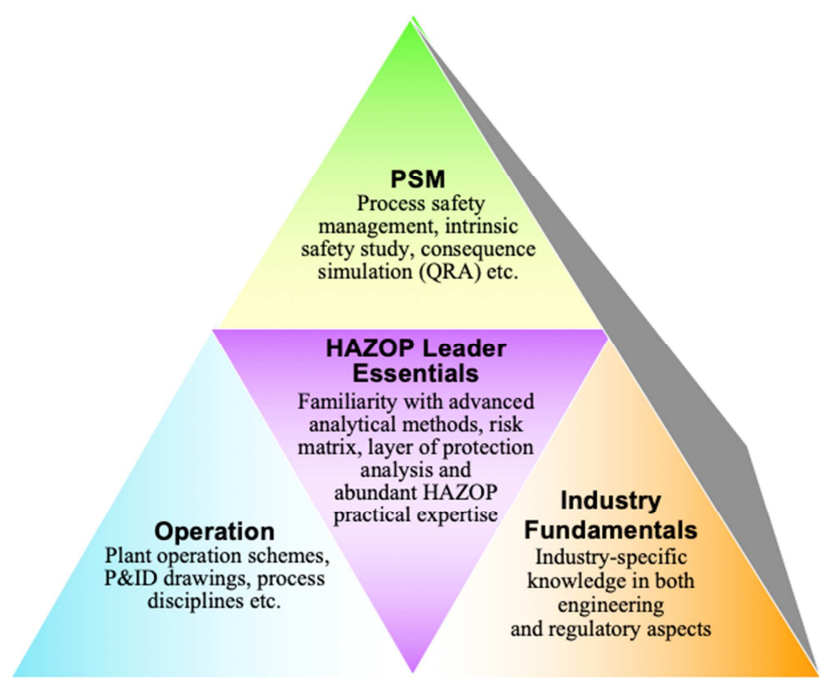

Figure 1. HAZOP Leadership Competency Model.

In practice, HAZOP chairman should be able to provide sufficient guidance and properly challenge with hypotheses to make sure all of the potential hazards are examined. Enough bandwidth should be allocated to analyzing significant but latent problems, instead of staying on those general and repetitive ones. Some international petrochemical companies sent their HAZOP experts to support the developing projects in other countries with consultancy and exchange the learnings globally. Another path is to cultivate internal HAZOP talents who are familiar with company technology, plant designs as well as HAZOP frameworks in order to lead the long-term corporate activities.

The contribution from multidisciplinary team members is 
equivalently critical to the outputs. A team should consist of designers, licensors, technical experts (on process operation, HSE, equipment and instrument) to have a thorough review with feedback from diverse perspectives. Meanwhile, it should not be neglected to keep a proper team size. Depending on the complexity and scale of projects, typically the HAZOP team keeps a size of 5-8 people to achieve an efficient and comprehensive discussion.

HAZOP process coordination is another important factor to the success of final outcomes. HAZOP chairman and team members need to cultivate an open atmosphere to inspire effective brainstorming that allows everyone to fully share their insights and concerns. Today more companies host their HAZOP conferences outside for a more concentrated discussion. HAZOP review is similar to a research process, and there should be an appropriate schedule with certain flexibility. Literature [7] showed improper schedule management is one of the common issues in industrial HAZOP activities in United States.

\subsection{Execution Strategy and Corporate HSE Culture}

The execution strategy covers the fundamentals of HAZOP analysis and project planning. While the overall corporate HSE culture nurtures the environment to achieve accurate and in-depth studies. The corporate should treat HAZOP analysis as a powerful tool in engineering management, not only to prevent hazards, but to improve the risk consciousness of all employees.

The following HAZOP/PHA fundamentals should be enhanced as routine practices:

Continuously improve risk management procedures with clear risk classification and the acceptable criteria.

Establish a quantitative risk analysis (QRA) database including typical chemical equipment and relevant failures, historical accident reports, abnormal state operation database etc.

Conduct periodical auditing to make sure HAZOP recommendations are executed in a thorough and efficient manner.

Develop a strong HAZOP committee in the long run by enhancing basic HAZOP trainings and cultivating talents with simulation mastery.

Set up a generic HAZOP template to streamline plant HAZOP analysis flow and boost efficiency.

Specific HAZOP plan should be developed based on the category and stage of different projects. For new or revamp plants, a detailed project plan should be in place covering all HAZOP activities in early period of the project [10]. Reasonable time nodes and overall budgeting should be well considered. In terms of execution, HAZOP progress should be incorporated into the overall project schedule to ensure a timely result delivery. For operating plants, periodic reviews should be performed to reduce process risks and resolve technical changes timely.

\section{Assurance Factors and Case Studies with Quantitative Approach}

The prerequisite factors involved in standard HAZOP process have been extensively discussed as above. This section mainly demonstrates how to supplement traditional HAZOP brainstorming by quantitative method based on accurate PSI data and simulation software. The factors in this section are named as assurance factors.

\subsection{HAZOP Design Data Accuracy and Systematicity [11]}

Consequence evaluation of hypothetical accident scenarios should leverage calculation, experiments, or other factual approaches with minimal assumptions, rather than staying at historical qualitative experiences.

For example, for a pressure relief system with safety valves and rupture discs, specific environment and process conditions need to be thoroughly examined; the safety interlocks, logic diagrams, and cause and effect table from licensors or design companies should be reviewed case by case, instead of relying on individual assumption. For the key physical and reaction parameters that are absent, it is recommended to perform pilot experiments for an accurate dataset to start with.

For instance, with semi-batch reactions, the reaction risk is typically determined by four important temperature parameters, i.e. process operation temperature $T_{p}$, maximum technical temperature MTT, temperature at the maximum reaction rate within $24 \mathrm{hrs} \mathrm{T}_{\mathrm{D} 24}$, and highest possible temperature of reaction MTSR. If a reaction has $T_{p}<T_{D 24}$ $<$ MTSR $<$ MTT, there will be high risk level with explosion hazards. The accurate temperature information should be obtained via reaction calorimetry or differential scanning calorimetry (DSC) to systematically predict the hazards and drive effective mitigations in advance [12].

\subsection{Incoporation of HAZOP Quantitative Approach}

Using process and accident consequence simulation to reach a convincing quantitative process hazard analysis (PHA) has become an inevitable trend for industry HAZOP development $[13,14]$.

HAZOP quantitative analysis is mainly associated with two aspects - process deviation simulation and risk quantification. Hereby we recommend three approaches commonly accepted in industry:

HAZOP risk matrix: risk matrix has been broadly applied to define the severity and probability of potential hazards in the past ten years. Recently more and more companies incorporated the Layer of Protection Analysis (LOPA) technique to evaluate the effectiveness of Independent Protection Layers (IPLs) and ensure the hazards under appropriate control $[15,16]$.

Accident consequence quantification: consequence simulation of explosions, fires and toxic leakage can be performed by quantitative risk analysis (QRA) or fault tree analysis (FTA) models [17, 18]. For impact range quantification on reactor explosion or leakage, industry software such as DNV·GL PHAST or SAFETI with pre-defined database can be a good option.

Process deviation quantification: for certain ambiguous 
risks either from process deviations or inadequate protection layers, it can sometimes exceed the scope of a single approach or software. An integrated computer-aided approach can be adopted by leveraging Aspen Plus, MATLAB and HYSYS simulation tools for optimized evaluation and solution development.

For instance, using Aspen Plus Dynamics to simulate the process of vacuum flash tower, assuming the gas control valve at the top of the tower was closed by human error, the tower pressure would reach 8.5 bar and $570^{\circ} \mathrm{C}$ in just 33 minutes if there were no protective measures. Based on the Aspen simulation, we'll be able to know an explosion accident can be caused here since the maximum allowable pressure and temperature was 2.8 bar and $449^{\circ} \mathrm{C}$ inside tower [19]. In another HAZOP example for an operating ethylene plant, the high-accuracy Operation Training Simulation system (OTS) developed by Honeywell was used to dynamically simulate the impact from key process deviations, such as the influence of charge gas compressor shutdown on the pipeline material of cold box outlet, and the excessive hydrogen impact on the temperature of $\mathrm{C} 2$ and $\mathrm{C} 3$ hydrogenation reactors. The depth and accuracy of HAZOP analysis can therefore be improved with quantitative simulation, leading to the development of effective protection layers and corrective actions.

\subsection{Accident Case Study with Semi-quantitative Method}

Applying HAZOP analysis with quantitative or half quantitative method makes the review of historical accidents more effectively. A real case study is presented below in demonstration of the practical value of HAZOP analysis in modern industry.

On March 23, 2005, an explosion and fire incident at the Isomerization (ISOM) Unit of BP Texas Refinery resulted in 15 fatalities and 170 injuries. This was one of the most severe accidents in US chemical industry over the past 30 years [20, 21].

Brief process of the accident: a liquid-overfill occurred at the raffinate splitter column which led to the release of the overhead relief valves. The overfilled liquid went through the discharge tank with an old-fashioned design (without containment system) then directly discharged into the atmosphere, ejecting large amount of flammable liquid that resulted in a massive fire and explosion accident.

Here we proposed a semi-quantitative approach of "HAZOP + LOPA + risk matrix" to review the accident. An $8 \times 8$ risk matrix was used to quantify the severity and probability of occurrence, in which "A-H" stands for the severity level from the strongest to the lightest; " $1-8$ " stands for occurrence from the least to the most frequent [22]. The LOPA technique was introduced to quantify the impact [23, 24] and identify the fundamental causes in order to develop thorough corrective actions.

Table 1 lists the identified causes, consequences, existing protective measures, and recommendations based on the semi-quantitative HAZOP analysis. The direct and systematical causes of the accident were correlated with the process deviations and effectiveness of safeguards as follows.

Table 1. HAZOP case study of 2005 BP Texas refinery incident by quantitative risk matrix.

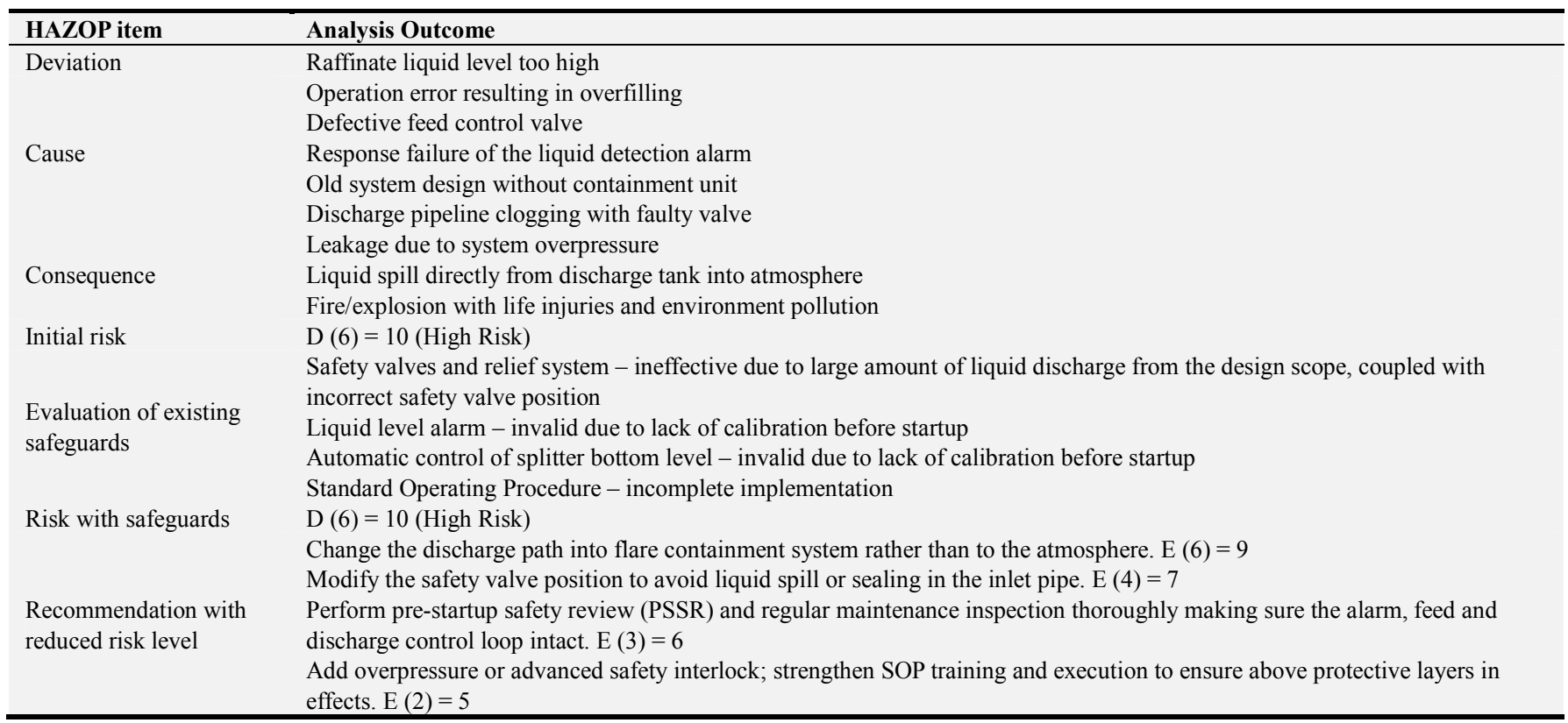

The critical causes were affirmed with the site investigation and analysis [21]:

The hazard of liquid emission was overlooked in the original Process Hazards Analysis (PHA), causing the lack of protective measures in the design.

The discharge tank was not connected to flare system, becoming a hidden hazard of the outlet.

Incorrect installation of safety valves resulted in fluid sealing up to 45 meters in the inlet pipe when splitter was full.

The startup procedures were not thoroughly executed, and the operation staff lacked sufficient trainings in crisis management. 
As shown in Table 1, since the discharge tank was directly open to the atmosphere, it had a fatality risk with more than 3 people (level D) - a high level risk. On top of that, the incorrect hazard evaluation of trailer office location and the improper control of onsite personnel and vehicles during startup also aggravated the impact of the incident.

If the outlet of discharge tank were connected to a flare containment system rather than directly into atmosphere, the highest risk level would have been reduced from $\mathrm{D}$ to $\mathrm{E}$ level with reduced fatality of 1-2 people. If all the recommendations were adopted, the risk level would be significantly reduced from D (6) $=10$ to $E(3)=6$ within the normal corporate risk range. As for whether it was necessary to add the safety interlock, it depends on the acceptable risk criteria of the specific company.

The above historical case study demonstrated that the impact of an accident can be significantly reduced with a thorough HAZOP analysis. Meanwhile quantitative approach played a critical role for better prioritization of risks and development of effective protective actions.

\section{Summary of Critical Factors on HAZOP Depth and Accuracy}

Ensuring the depth and accuracy of HAZOP analysis is the most important yet difficult task in the entire process. Aiming at improving the overall quality of industrial HAZOP analysis, this study elaborated the critical factors and countermeasures from prerequisite and assurance aspects. Comprehensive identification of process hazards and effective protective layers would prevent incidents from happening and greatly minimize the impact.

To better highlight the takeaways of the study, Figure 2 presents an intuitive illustration for future reference and implementation.

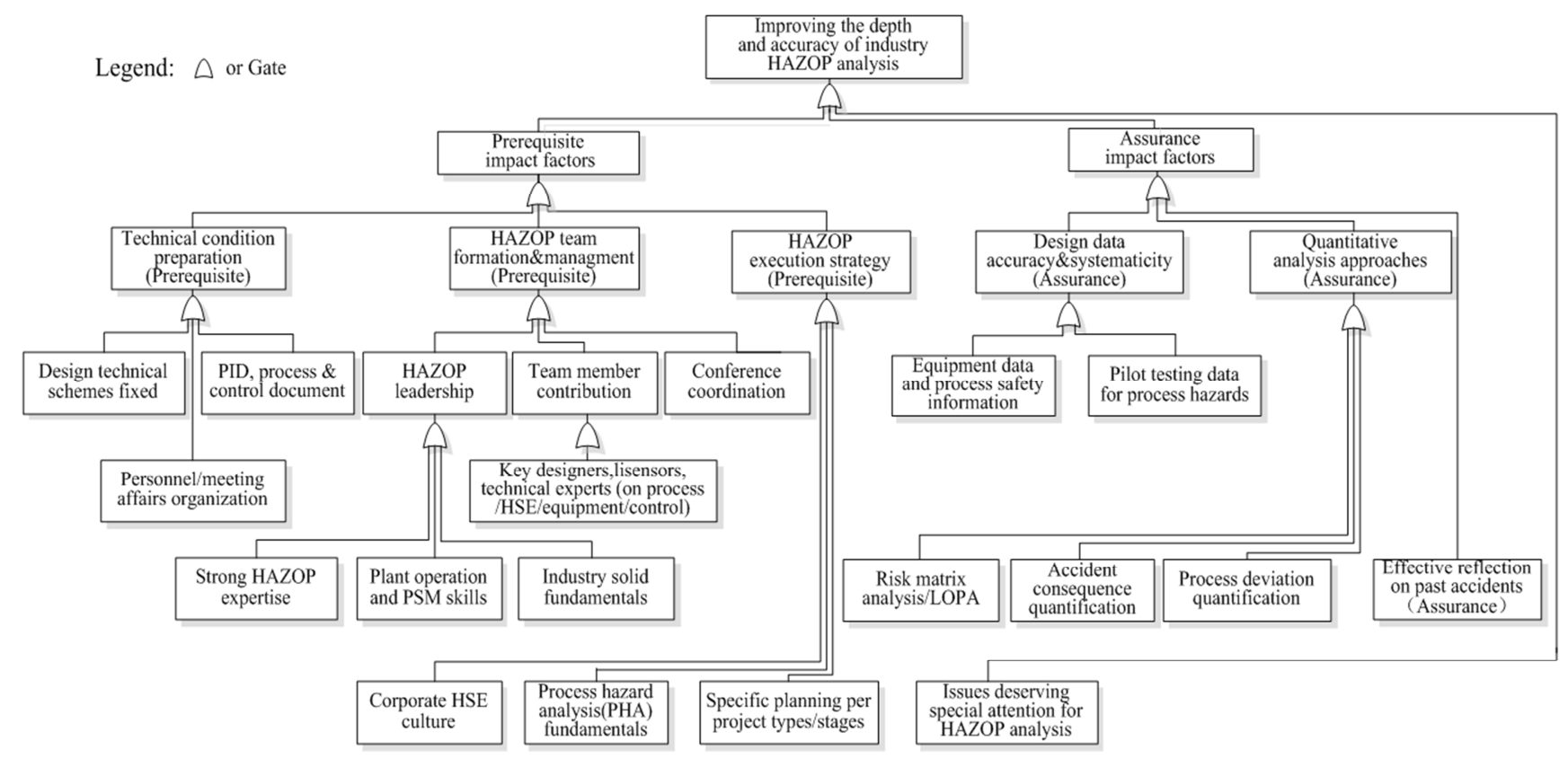

Figure 2. Overview of critical impact factors on the depth and accuracy of HAZOP analysis.

\section{Several Issues Deserving Special Attention}

To deliver in-depth and accurate HAZOP outcomes, there are several issues easily to get overlooked and deserving particular attention below.

\subsection{Effectiveness of the safeguards}

It is necessary to ensure the safeguards are effective before closure of HAZOP analysis. For a specific deviation or scenario, confirmation on the effectiveness of current protective measures is a key step yet easy to get misused in HAZOP activities. Examples are such as the check valves without regular inspection, the alarms without sufficient time for response, safety interlocks not independent from process control, and the gap in SOP implementation. All of these protective measures are considered ineffective. The acceptable risk level and risk management principles vary with different enterprises. It is a good practice for a company to establish its own risk regulations and incorporate them into formal HAZOP procedures for consistent execution [25].

\subsection{Coming Up with Recommendations}

Engineering recommendations should be provided if any HAZOP concern is identified associated with situations below:

Significant hazard or process impact

Insufficient safeguards or administrative controls to mitigate the consequence

No safety risk but a severe operability concern 
Shortfall in compliance with industry standards or company regulations

Make sure key components are included when drafting the recommendations: 1) clear problem defintion and technical background underlining significance of the problem 2) be concise but specific about the details like equipment and piping numbers 3) specify the location that needs modification and the feasible proposals aligned by the team with projected timeline.

\section{Conclusion}

This study reviews the significance and existing issues of HAZOP analysis in modern industries. Conventional HAZOP technique has been used as the qualitative risk evaluation approach in past decades, which largely depends on historical experience or brainstorming and may lead to inaccurate hazard identification and severe accident impact. This article aims at improving the depth and accuracy of HAZOP analysis, by highlighting critical impact factors and proposing quantitatively integrated framework with the lessons drawn on classical industrial cases.

The presented study systematically analyzes two types of impact factors, namely prerequisite factors and assurance factors. The former consists of technical conditions, HAZOP team management, execution strategy and HSE Culture, which are the fundamentals of HAZOP analysis. While the latter is associated with systematical PSI data, quantitative analytical frameworks, and effective accident review for long-term HAZOP optimization. A thorough case investigation using semi-quantitative method (i.e. risk matrix and LOPA elements) was illustrated.

For future research, application issues can be explored regarding efficiency improvement and resource allocation in order to yield the best outcomes with minimal time investment. Meanwhile finer study can be performed on different kinds of projects from newly built to special revamp ones in order to develop targeted HAZOP strategies. It should also be emphasized that the HAZOP framework should serve more as a problem-solving concept rather than just the specific tool. Thus, it would be an impactful direction to apply HAZOP methodology into other industries to address broader challenges.

Through management of the critical factors and integrated quantitative approach, the influence of enterprise HAZOP analysis will be more profound with enhanced accident prevention and risk awareness in the overall industrial environment.

\section{References}

[1] Occupational Safety and Health Administration (OSHA). OSHA PSM (29 CFR 1910.119), Process Safety Management of highly hazardous chemicals. 29 CFR Ch. XVII (7-1-99 Edition).

[2] Cagno, E., F. Caron, et al. Risk analysis in plant commissioning: the Multilevel HAZOP. Reliability Engineering \& System Safety. 2002, 77, 309-323. [DOI: 10.1016/S0951-8320(02)00064-9]

[3] Dunjó, J.; Fthenakis, V.; Vílchez, J. A.; Arnaldos, J. Hazard and Operability (HAZOP) Analysis. A literature review. $J$. Hazard. Mater. 2010, 173, 19-32. [DOI: 10.1016/j.jhazmat.2009.08.076]

[4] Li, Y. J. The exploration of integrity assurance of project HAZOP analysis and adoption extension of its method. $J$. Modern Chemical Industry, 2019, 39, 11-15.

[5] Cheng, Xi'an; Zhang, Q. Research and application of HAZOP deviation quantization based on process simulation. J. Modern Chemical Industry. 2017, 37, 187-191.

[6] Baybutt, P. A critique of the Hazard and Operability (HAZOP) study. J. Loss Prev. Process. Ind. 2015, 33, 52-58. [DOI: 10.1016/j.jlp.2014.11.010]

[7] Common Mistakes When Conducting a HAZOP and How to Avoid Them. Article in Chemical Engineering, New York, Mcgraw Hill Incorporated then Chemical Week Publishing 1lc-122 (12): 54-58 December 2015.

[8] Baybutt, P. On the completeness of scenario identification in process hazard analysis (PHA). J. Loss Prev. Process. Ind. 2018, 55, 492-499. [DOI: 10.1016/j.jlp.2018.05.010]

[9] Rossing, N. L.; Lind, M.; Jensen, N.; Jorgensen, S. B; A Goal based methodology for HAZOP analysis, Published in, J. International journal of nuclear safety and simulation. 2010, 1, 133-142.

[10] Aminbakhsh, S.; Gündüz, M.; Sonmez, R. Safety risk assessment using analytic hierarchy process (AHP) during planning and budgeting of construction projects. J. Saf. Res. 2013, 46, 99-105.

[11] Rossing, N. L. Method development for systematic risk assessment, Kgs. Lyngby: Technical University of Denmark Dept. of Chemical Engineering, M. Sc. Thesis, 2006.

[12] Zhai, Q. W., Tang, B. How to apply chemical reaction heat data to HAZOP analysis and engineering practice protection. The 4th China International Chemical Process Safety Conference, Suzhou. 2019.11.

[13] Chen, Y.; Lai, X. L. The latest development of HAZOP analysis method and its application. J. Petrochemical Safety and Environmental Technology. 2013, 29, 30-34.

[14] Venkatsubramanian, V.; Zhao, J.; Viswa, N. S. Intelligent systems for HAZOP analysis of complex process plants, computers chemical engineering, 2000, 24, 2291-2302. [DOI: 10.1016/S0098-1354(00)00573-1]

[15] Marhavilas, P. K.; Filippidis, M.; Koulinas, G. K.; Koulouriotis, D. E. The integration of HAZOP study with risk-matrix and the analytical-hierarchy process for identifying critical control-points and prioritizing risks in industry-A case study. J. Loss Prev. Process. Ind. 2019.

[16] Trammell, S. R.; Lorenzo, D. K.; Davis, B. J. Integrated hazards analysis-using the strengths of multiple methods to maximize effectiveness. J. Professional Safety. 2004, 49, 29 37.

[17] Feng, Y.; Ma, K.; Liao, Z. G. PHAST- based Leakage Simulation of Propylene Sphere. J. Safety, Health and Environment. 2015, 15, 46-49. 
[18] Ozog, H.; Bendixen, L. M. Hazard identification and quantification: the most effective way to identify, quantify, and control risks is to combine a hazard and operability study with fault tree analysis. J. Chemical Engineering Progress. 1987, 83, 55-64.

[19] Yan, W. Q.; Yun, Y. T.; Qi, M.; Zhao, D. F. Quantitative HAZOP Analysis and Research Based on Aspen. Collected Papers of the Sixth CCPS China Process Safety Conference 999-1007. Qingdao, 2018.9.

[20] James, A. B.; Nancy, L.; Frank L.; Skip, B.; et al. BP American Refinery Independent Safety Review Team Report.

[21] MOGFORD, J.: Fatal accident investigation report, Texas City: BP, 2005

[22] Wu. H. J. Application of Risk Matrix in Liquid Ammonia Transportation Mode Decision. J. Safety Health \&Environment. 2017, 17, 41-44.
[23] Alaei, R.; Mansoori, S. A. A.; Moghaddam, A. H.; Mansoori, S. M.; Mansoori, N. Safety assessment approach of hazard and operability (HAZOP) for sulfur recovery unit Claus reaction furnace package; blower; heat exchanger equipment in South Pars gas processing plant. J. Nat. Gas Sci. Eng. 2014, 20, 271-284. [DOI: 10.1016/j.jngse.2014.07.007].

[24] Demichela, M.; Marmo, L.; Piccinini, N. Recursive operability analysis of a complex plant with multiple protection devices. J. Reliability Engineering and System Safety. 2002, 77, 301-308. [DOI: 10.1016/S0951-8320(02)00063-7].

[25] Hurme, M; Rahman, M. Implementing inherent safety throughout process lifecycle. J. Journal of loss prevention in the Process Industries. 2005, 18, 238-244. [DOI: 10.1016/j.jlp.2005.06.013]. 